\title{
Safety and efficacy of combined antegrade and retrograde endoscopic dilation for complete esophageal obstruction: a systematic review and meta-analysis
}

\author{
Mahendran Jayaraja*, Babu P. Mohan ${ }^{\text {b*, }}$, Harmeet Mashianac, Rajesh Krishnamoorthid, Douglas G. Adler \\ University of Nevada, Las Vegas; University of Arizona, Banner University Medical Center, Tuscon, Arizona; University \\ of Nebraska Medical Center; Digestive Diseases Institute, Virginia Mason Medical Center, Seattle, Washington; \\ University of Utah, School of Medicine, Salt Lake City, Utah, USA
}

\section{Abstract}

${ }^{a}$ Department of Gastroenterology and Hepatology, University of Nevada Las Vegas School of Medicine (Mahendran Jayaraj);

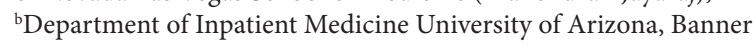
University Medical Center, Tucson, Arizona (Babu P. Mohan); 'Department of Gastroenterology and Hepatology University of Nebraska Medical Center, Omaha, Nebraska (Harmeet Mashiana); ${ }^{\mathrm{d} D e p a r t m e n t ~ o f ~ G a s t r o e n t e r o l o g y ~ D i g e s t i v e ~ D i s e a s e s ~ I n s t i t u t e, ~ V i r g i n i a ~}$ Mason Medical Center, Seattle, Washington (Rajesh Krishnamoorthi); ${ }^{\mathrm{e} D i v i s i o n}$ of Gastroenterology and Hepatology, University of Utah School of Medicine, Salt Lake City, Utah (Douglas G. Adler), USA

* Authors contributed equally to the manuscript

DOI: https://doi.org/10.20524/aog.2019.0385
Conflict of Interest: None

Correspondence to: Douglas G. Adler MD, FACG, AGAF, FASGE, Professor of Medicine, Director of Therapeutic Endoscopy, Directory, GI fellowship Program, Gastroenterology and Hepatology, University of Utah School of Medicine, Huntsman Cancer Center, 30N, Salt Lake City, Utah 84132, USA, e-mail: Douglas.adler@hsc.utah.edu

Received 21 December 2018; accepted 6 February 2019; published online 16 May 2019 


\section{Introduction}

Complete esophageal obstruction (CEO) is rare and usually occurs after radiotherapy in head and neck cancer patients, but it can arise in several other clinical settings, such as gastroesophageal reflux disease, caustic ingestion and Plummer-Vinson syndrome [1,2]. Antegrade reopening and dilation of a CEO is difficult and carries a high risk of esophageal perforation [3]. A combined antegrade-retrograde rendezvous procedure with recanalization and dilation (CARD) may offer better visualization and potentially safer dilation [4]. This procedure was first described by van Twisk et al [5] in 1998, followed by Bueno in 2001 [4].

The basic principle of CARD is to endoscopically reach the proximal and distal ends of the stricture to better control the dilation. Patients are usually under general anesthesia. An upper endoscopy determines the upper level of stricture by direct visualization. The gastroesophageal junction is visualized and entered with an endoscope via the gastrostomy tract after appropriate dilation of the tract. The endoscope is advanced to the level of the stricture. A guidewire is pushed through the stricture under fluoroscopy and grasped by the upper endoscope. Once pulled through the mouth, the guidewire is used as a guide to insert dilators sequentially in antegrade fashion to the desired diameter.

Many case reports and small sized studies have reported on the technical aspects of the procedure [4,6-23]. Only a handful have reported on the actual clinical outcomes, such as swallowing, percutaneous endoscopic gastrostomy (PEG)-tube dependency and/or freedom and the need for repeat dilation [8-10,14]. An overall estimate of the outcomes with CARD for CEO is not known, because there is a paucity of large, good-quality studies. Therefore, we conducted this systematic review and meta-analysis to better understand the clinical outcomes, the technical success, and the safety of the procedure.

\section{Materials and methods}

\section{Search strategy}

We conducted a comprehensive search of several databases and conference proceedings, including PubMed, EMBASE, and Web of Science databases (earliest inception to November 2018). We followed the Preferred Reporting items for Systematic Reviews and Meta-Analyses (PRISMA) guidelines [24], using a predefined protocol to identify studies reporting CARD for CEO.

The key words used in the search included a combination of combined antegrade retrograde endoscopic dilation, combined antegrade retrograde dilation, complete esophageal obstruction, esophagus, CARD, CEO, success, adverse events and complications in various combinations to identify original published studies. The search was restricted to studies in human subjects that were published in the English language in peerreviewed journals. Two authors (MJ, HM) independently reviewed the titles and abstracts of studies identified in the primary search and excluded studies that did not address the research question, based on pre-specified exclusion and inclusion criteria. The full text of the remaining articles was then reviewed to determine whether it contained relevant information. Any discrepancy in article selection was resolved by consensus and in discussion with a co-author (RK). The bibliographic sections of the selected articles, as well as the systematic and narrative articles on the topic, were manually searched for additional relevant articles.

\section{Study selection}

In this meta-analysis, we included cohort studies that met the following criteria: 1) CARD procedure for CEO; 2) data available on technical success, clinical success in terms of dysphagia improvement, and freedom from PEG tube (defined as removal of PEG tube); and 3) data on adverse events. In order to increase the number of studies available for analysis, studies were also included irrespectively of the geography, abstract/ manuscript status and initial radiotherapy treatment data, as long as they provided the primary data needed for the analysis. Our exclusion criterion was single procedure case reports.

In case of multiple publications from the same cohort, data from the most recent comprehensive report would be included. However, we did not encounter any such studies in our selection process.

\section{Data abstraction and quality assessment}

Data on study-related characteristics as well as reported outcomes in the individual studies were abstracted onto a standardized form by at least 2 authors (MJ, BPM) independently. The quality of the studies was assessed using the Newcastle-Ottawa scale for cohort studies [25]. This quality score consisted of 7 questions: representative of the average adult in the community ( 1 point for population-based studies, 0.5 point for multi-center studies; 0 points for a single-center hospital-based study); large cohort size ( 1 point if $>20$ cases, 0.5 point if between 10 and 20 cases, 0 points if $<10$ cases); information on technical success (1point if reported; 0 points if not reported); information on clinical success ( 1 point if reported, 0 points if not reported); information on adverse events (1 point if reported, 0 points if not reported); type of article write-up ( 1 point if original manuscript, 0.5 point if abstract); and attrition rate ( 1 point if all CARD-CEO cases accounted for, 0.5 point if $<50 \%$ cases not accounted for, 0 points if $>50 \%$ cases not accounted for). Scores of $>5,3$ to 5 , and $<3$ were considered suggestive of highquality, medium-quality, and low-quality studies, respectively.

\section{Outcomes assessed}

The primary analysis focused on assessing the outcomes of the CARD procedure. The outcomes measured and analyzed were: 1) technical success; 2) clinical success in terms of dysphagia improvement, PEG-tube dependence, PEG-tube free; and 3 ) the need for repeated dilation. The secondary 
outcomes assessed were complications from the CARD procedure: 1) pneumomediastinum; 2) perforation; and 3) death. Subgroup analysis was performed for studies with sample size $>5$ patients and $<5$ patients.

\section{Statistical analysis}

We used meta-analysis techniques to calculate the pooled estimates in each case, following the methods suggested by DerSimonian and Laird [26], using the random-effects model. When the incidence of any effect was zero in a study, a correction of 0.5 was added to the number of incident cases before statistical analysis [27]. We assessed heterogeneity between study-specific estimates using two methods [28,29]. First, the Cochran Q statistical test for heterogeneity was performed, which tests the null hypothesis that all studies in a meta-analysis have the same underlying magnitude of effect. Second, when heterogeneity was present, in order to estimate what proportion of total variances across studies was due to heterogeneity rather than chance, the $I^{2}$ statistic was calculated. In this, values of $<30 \%$, $30-60 \%, 61-75 \%$ and $>75 \%$ were suggestive of low, moderate, substantial and considerable heterogeneity, respectively [30]. Since the random-effects model estimates an average effect, we also calculated the $95 \%$ prediction interval, which deals with the dispersion of the effects [31]. Publication bias was ascertained, qualitatively by visual inspection of a funnel plot and quantitatively using Egger's test of the intercept [32]. All analyses were performed by using Comprehensive MetaAnalysis software, version 3 (BioStat, Englewood, NJ).

\section{Results}

\section{Search results and population characteristics}

From a total of 516 citations identified using our search strategy, 36 studies reported clinical success, technical success and adverse events in patients undergoing CARD for CEO. Of these, 17 studies were excluded because they did not meet the study criteria. 19 studies [4,6-23] were included in the final analysis. A schematic diagram of the study selection is provided in Fig. 1.

The majority of the studies were from the USA. The analysis included a total of 229 patients who underwent a total of 251 CARD procedures. The mean age ranged from $56-78.5$ years. In $79 \%$ of the cases the $\mathrm{CEO}$ was due to prior radiotherapy. Other less common causes were gastroesophageal reflux disease, PlummerVinson syndrome, and caustic injury. Dysphagia was assessed in different ways, as follows: Dakkak and Bennet scores, swallow therapist assessment, and functional oral intake scale levels.

\section{Characteristics and quality of included studies}

Table 1 presents the characteristics of the included studies and Supplementary Table 1 shows the quality of the included studies. All studies reported single-center data. All of the studies had definite information on the use of CARD in CEO cases. All studies reported adequately on the technical success. Three studies $[11,13,17]$ did not provide complete information on the clinical success. We included 3 abstracts $[8,14,16]$, while the remainder were full manuscripts. Overall, 4 studies [8-10,14] were considered high quality and the rest were considered medium quality. None were of low quality.

\section{Primary and secondary outcomes}

All results, along with a subgroup analysis of studies with $>5$ patients, are summarized in Table 2 . The pooled rate of technical success was $88.9 \%$ (95\% confidence interval [CI] $83.9-92.5, l^{2}=0$ ) (Fig. 2). This means that $88.9 \%$ of patients who underwent CARD had the obstruction traversed. The pooled rate of dysphagia improvement was 58.4\% (95\%CI 50-66.3, $I^{2}=12.6$ ). This means that approximately $40 \%$ of patients had no improvement in dysphagia and needed alternative management, such as repeat dilation or PEG-tube feeding, even though the CARD procedure was successful. This can be seen from the fact that the pooled rate of being PEG-tube free was $43.5 \%$ (95\%CI 34.1-53.4, $I^{2}=30.6$ ), while the pooled rate of being PEG-tube dependent was $41.5 \%\left(95 \%\right.$ CI $\left.32.7-50.8, I^{2}=32\right)$. The pooled rate of repeat dilations after CARD was $78.9 \%$ (95\%CI 69.7-85.8, $I^{2}=15.2$ ). Subgroup analysis of the studies based on the sample size of $>5$ and $<5$ showed that clinical outcomes, including dysphagia improvement $81.9 \%$ (95\%CI 57.5-93.8, $\left.I^{2}=0\right)$ and PEG-tube freedom $72.6 \%$ (95\%CI 35.5-92.8, $I^{2}=24$ ), were slightly superior in studies with less than 5 patients (Fig. 3, and Suppl. Figs. 1-3).

The pooled rate of perforation was $8 \%\left(95 \% \mathrm{CI} 4.8-13, I^{2}=0\right)$ and that of pneumomediastinum was $9.9 \%$ (95\%CI 6.2-15.6, $I^{2}=0$ ). Subgroup analysis showed that the rates were higher in studies with less than 5 patients: perforation 10.9\% (95\%CI 3.2$\left.31.4, I^{2}=0\right)$ and pneumomediastinum $16.9 \%$ (95\%CI 5.5-41.5, $I^{2}=0$ ). This could be a small study effect on the meta-analysis, which is why we did the subgroup analysis (Suppl. Fig. 4 and 5).

The pooled rate of death was $6.8 \%\left(95 \%\right.$ CI $\left.3.4-13.1, I^{2}=0\right)$, although the actual reported number of deaths was 1 , not procedure-related (Suppl. Fig. 6).

\section{Sensitivity analysis and publication bias}

To assess whether any one study had a dominant effect on the meta-analysis, we excluded one study at a time and analyzed its effect on the main summary estimate and heterogeneity. According to this analysis, no single study significantly affected the primary outcome. Based on a visual inspection of the funnel plot as well as quantitative measurement using the Egger regression test $(\mathrm{P}=0.27)$, there was no evidence of publication bias (Suppl. Fig. 7).

\section{Discussion}

Our study is the first meta-analysis to report on the outcomes of the CARD procedure in the management of CEO. 
Based on our analysis, we report an overall technical success rate of $89 \%$. CARD is not a new concept and has been used for the open surgical treatment of distal esophageal stenosis during anti-reflux procedures [4]. In most cases, patients are too frail to undergo surgery and are usually considered suitable for CARD. The most difficult part of the procedure is gaining access through the completely obstructed esophagus and the chances of recurrence are high [13].Repeat dilations are often needed when symptoms recur. Based on our study, the pooled rate of repeat dilation after CARD was $79 \%$. This high rate can be partly explained by the complex nature of the causes for CEO. The major cause of CEO from the studies reviewed was radiotherapy for head and neck cancer[1,2,24,25]. The other well-known causes are caustic ingestion, chronic reflux, and esophageal surgery [24,25].
We assessed the clinical outcomes of CARD for CEO by calculating the pooled rates of dysphagia improvement, being PEG-tube free and being PEG-tube dependent. We report a dysphagia improvement rate of $58.4 \%$, albeit with some variability in the methodology used to report the improvement. This was reported to be $44 \%$ in the largest case series to date by Grooteman et al [9]. They used the Dakkak and Bennet score [26] to assess improvement in swallowing. This score is a combination of subjective and objective parameters based on patients' accounts of their eating capacity and their observed performance. Goguen et al [22] reported dysphagia improvement in terms of achieved diet as recorded by swallow therapists, whereas Bertolini et al [20] used the functional oral intake scale [27]. Patients who failed to show a successful clinical outcome have few other options to choose from other than

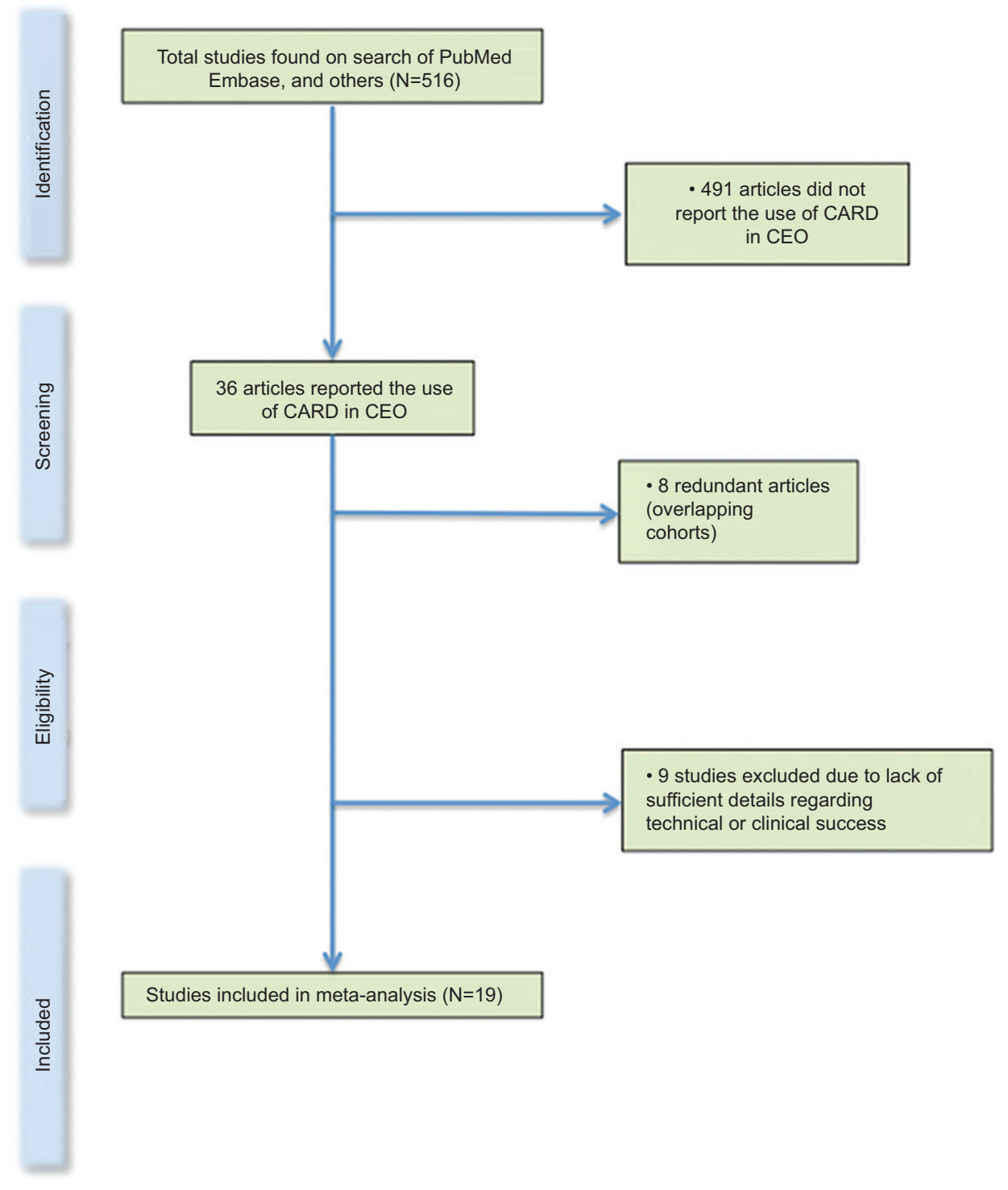

Figure 1 Study flow and section $C A R D$, combined antegrade and retrograde endoscopic dilation; CEO, complete esophageal obstruction 
Safety and efficacy of CARD for CEO 5

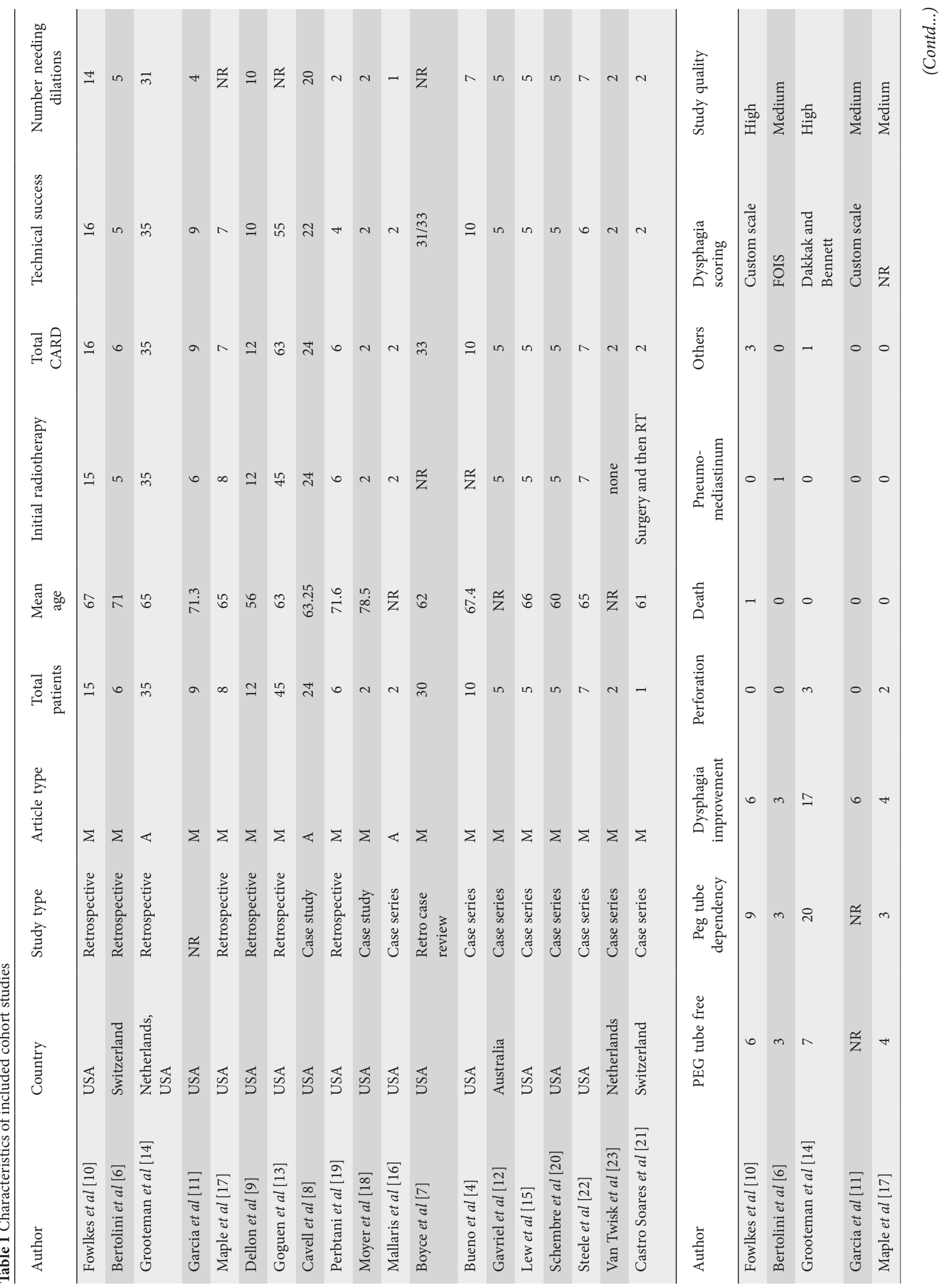




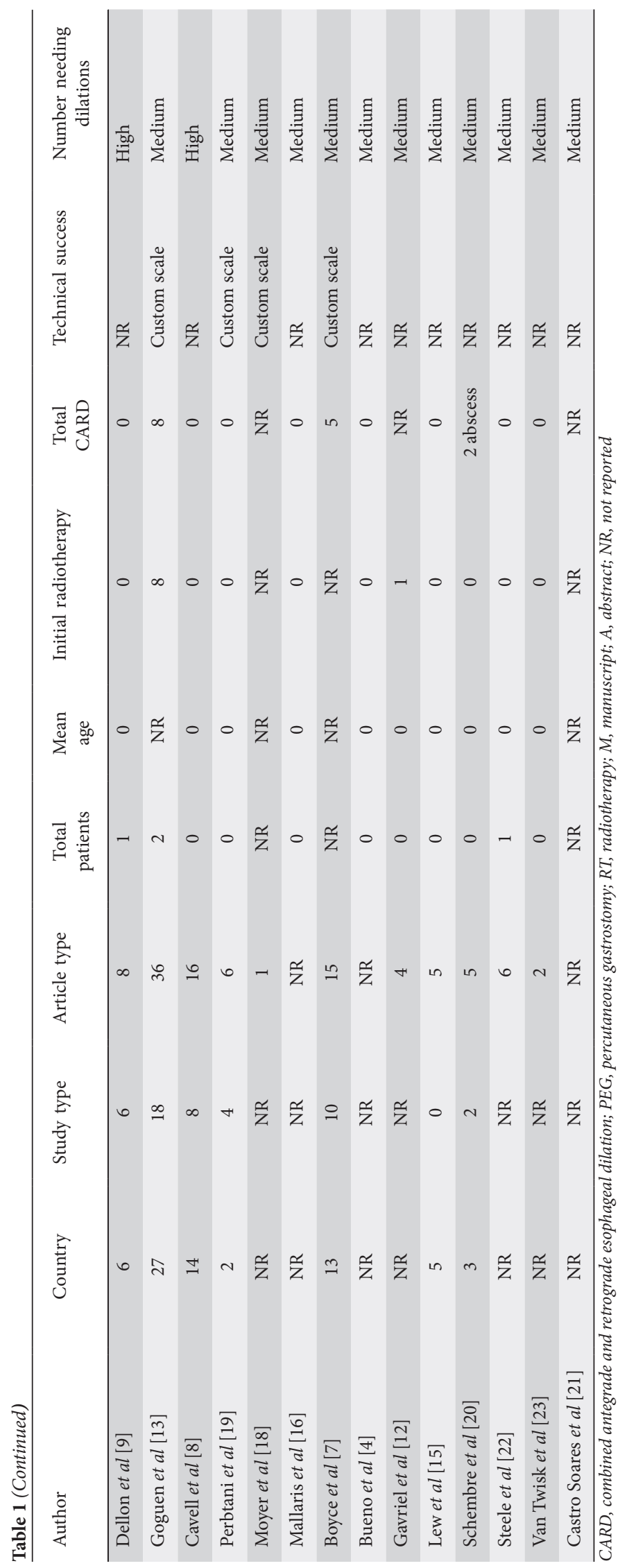


being on a PEG-tube to meet nutritional needs. These include a novel technique called per-oral endoscopic tunneling for the restoration of the esophagus (POETRE) and/or placement of a stent [33]. Partially covered, fully covered and biodegradable stents have been used to this end [34]. A multicenter series studying the natural history and management of refractory benign esophageal strictures showed that the long-term outcomes with serial balloon dilations and/or stent placement appear to be suboptimal, with only one third achieving clinical resolution, defined as no need for endoscopic interventions for at least 6 months [35]. The role of stent placement for refractory benign esophageal strictures is not well established. A metaanalysis by Fuccio et al shows that stent placement was effective in only $40 \%$ of patients [34]. A recent randomized controlled trial evaluated the outcomes comparing serial balloon dilation and placement of biodegradable stents for benign esophageal strictures and reported fewer interventions in the stented group at 3 months, albeit with similar outcomes at 6 months [36].

Based on our analysis, we report a PEG-tube free rate of 43.5\% and a PEG-tube dependency rate of $41.5 \%$. The reported data on the utilization of PEG-tube after CARD varied among the studies. Although a PEG-tube free outcome would mean taking food orally, studies did report patients who were being fed by mouth and at the same time were using the PEGtube to meet their daily nutrition goal. A component of the reported PEG-tube dependent patients were from technically unsuccessful cases. In other words, we were unable to directly

Table 2 Results

\begin{tabular}{lccc}
\hline Parameter & Overall & Sample size $>5$ & Sample size $<5$ \\
& & $\left(95 \%\right.$ CI, $\left.I^{2}\right)$ & $87.7 \%$ \\
& & $89.1 \%$ & $(69.9-95.6,0)$ \\
& & $(83.7-92.9,0)$ & $81.9 \%$ \\
Technical success & $88.9 \%$ & $55.3 \%$ & $(57.5-93.8,0)$ \\
Dysphagia improvement & $58.9-92.5,0)$ & $(48.4-61.9,9.5)$ & $72.6 \%$ \\
PEG free & $(50-66.3,12.6)$ & $41.5 \%$ & $(35.5-92.8,24)$ \\
& $43.5 \%$ & $(33.1-50.3,22.9)$ & $28.3 \%$ \\
PEG dependent & $(34.1-53.4,30.6)$ & $42.5 \%$ & $(7.2-66.8,4.8)$ \\
& $41.5 \%$ & $(33-52.5,39.6)$ & $84.6 \%$ \\
Repeat dilation & $(32.7-50.8,32)$ & $77.3 \%$ & $(63.4-94.5,0)$ \\
& $78.9 \%$ & $(66-85.7,46.8)$ & $10.9 \%$ \\
Perforation & $(69.7-85.8,15.2)$ & $7.5 \%$ & $(3.2-31.4,0)$ \\
& $8 \%$ & $(4.3-12.8,0)$ & $16.9 \%$ \\
Pneumo-mediastinum & $(4.8-13,0)$ & $8.9 \%$ & $(5.5-41.5,0)$ \\
& $9.9 \%$ & $(5.3-14.6,0)$ & $14.1 \%$ \\
Death & $(6.2-15.6,0)$ & $5 \%$ & $(4.1-38.9,0)$ \\
\hline
\end{tabular}

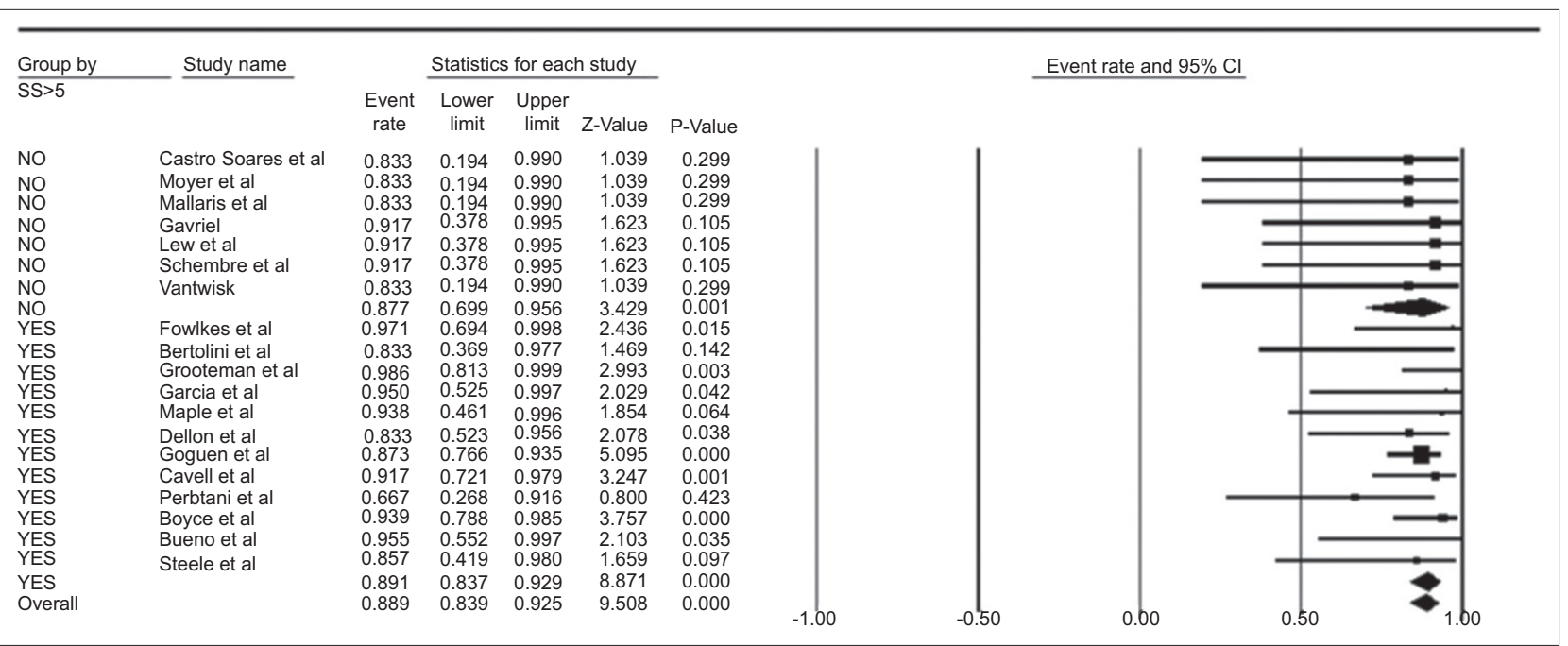

Figure 2 Forest plot. Technical success of combined antegrade and retrograde endoscopic dilation in omplete esophageal obstruction by sample size (SS) $<5$ patients and $>5$ patients

CI, confidence interval 


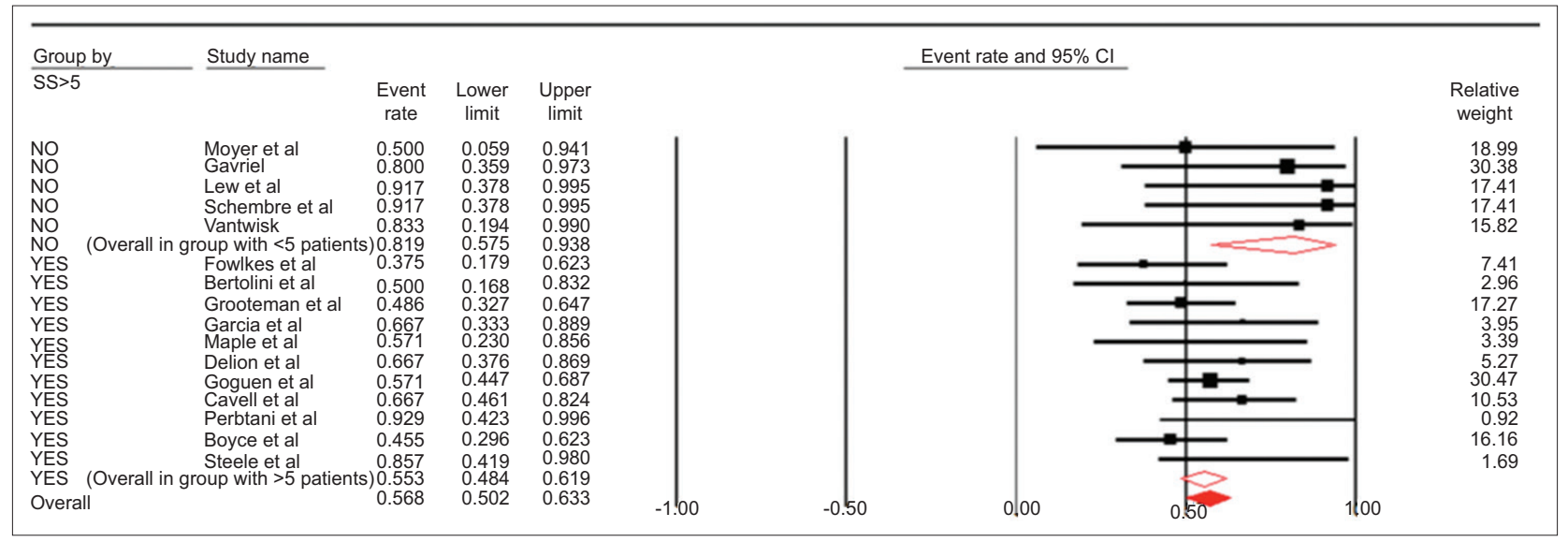

Figure 3 Forest plot. Dysphagia improvement by sample size (SS) $<5$ patients and $>5$ patients CI, confidence interval

attribute the PEG-tube outcomes to the technically successful CARD procedures.

In our analysis of adverse events, the most commonly reported ones were perforation, pneumomediastinum, and death. The pooled rate of perforation was $8 \%$ and the pooled rate of pneumomediastinum was about $10 \%$. These adverse events are very important, as they carry a high morbidity and mortality potential in an already frail patient. The pooled rate of death was $6.8 \%$ and this value needs to be interpreted with caution. Only one death event was reported from the included studies and it was not associated with the CARD procedure, but was rather due to the underlying disease process. Our calculated value seems high as a result of the 0.5 continuity correction applied to the outcomes that were zero.

The strengths of this review are as follows: systematic literature search with well-defined inclusion criteria; carefully excluding redundant studies; detailed extraction of overall data in terms of technical and clinical success outcomes; extraction of data on adverse events; rigorous evaluation of study quality; and robust statistics to accept and/or refute our findings. Low heterogeneity and absence of publication bias positively influence the validity of our analysis. We subgrouped our outcomes based on the sample size (total patients $<5$ and total patients $>5$ ). Considerable variation between these groups was observed in the calculated rates of clinical success. In this way, we hoped to demarcate the effects of small-size studies on the reported outcomes of an uncommon procedure in a rare disease entity. Thus, our metaanalysis results are an important addition to the current literature.

Our study has limitations, most of which are inherent to any meta-analysis. Our included studies had many retrospective reviews that added to selection bias. Although the included studies had good data on technical success, they varied in their reports of clinical outcomes. The definitions of being PEG-tube dependent and being PEG-tube free were not uniform across the studies. There is no standard scale for measuring dysphagia improvement and different measures were used in the included studies. Moreover, the included studies were not entirely representative of the general population and community practice. However, this estimate is still the best available estimate and may be used in predicting clinical outcomes and counseling patients on the CARD procedure. We were unable to identify a high-risk subset of patients and risk-stratify the reported outcomes. The current literature is clearly limited and more large-scale prospective studies are needed to evaluate the CARD procedure in CEO cases. Stents with biodegradable and lumen-apposing properties seem to show promising results in the management of gastrointestinal strictures.

In conclusion, we determined that the CARD procedure in cases of CEO has a technical success rate of $90 \%$, with a $79 \%$ repeat dilatation rate. Less than half of the patients would be expected to

\section{Summary Box}

\section{What is already known:}

- A combined antegrade and retrograde rendezvous procedure with recanalization and dilation (CARD) offers better visualization and safer dilation of complete esophageal obstruction (CEO)

- Outcomes of CARD in CEO have been reported from a few small-sized studies; therefore, an overall good-quality estimate of this procedure is not known

\section{What the new findings are:}

- Estimated technical success of CARD in CEO is $88.9 \%$

- After a CARD procedure for CEO, the estimated dysphagia improvement is $58.4 \%$, being PEG-tube free is $43.5 \%$, and being PEG-tube dependent is $41.5 \%$

- Estimated rate of repeat dilations after CARD in CEO is $78.9 \%$

- Estimated pooled rate of perforation with the procedure is $8 \%$ and pneumomediastinum is about $10 \%$ 
remain PEG-dependent and a majority will improve with regard to their ability to swallow. Based on our reported adverse events, the procedure should be offered after taking into consideration patient characteristics, endoscopist experience, and availability of resources.

\section{References}

1. Coia LR, Myerson RJ, Tepper JE. Late effects of radiation therapy on the gastrointestinal tract. Int J Radiat Oncol Biol Phys 1995;31:1213-1236.

2. Laurell G, Kraepelien T, Mavroidis P, et al. Stricture of the proximal esophagus in head and neck carcinoma patients after radiotherapy. Cancer 2003;97:1693-1700.

3. Banerjee A, Rao KS, Nachiappan M. Intrathoracic oesophageal perforations following bougienage: a protocol for management. Aust N Z J Surg 1989;59:563-566.

4. Bueno R, Swanson SJ, Jaklitsch MT, Lukanich JM, Mentzer SJ, Sugarbaker DJ. Combined antegrade and retrograde dilation: a new endoscopic technique in the management of complex esophageal obstruction. Gastrointest Endosc 2001;54:368-372.

5. van Twisk JJ, Brummer RJ, Manni JJ. Retrograde approach to pharyngo-esophageal obstruction. Gastrointest Endosc 1998;48:296-299.

6. Bertolini R, Meyenberger C, Putora PM, et al. Endoscopic dilation of complete oesophageal obstructions with a combined antegrade-retrograde rendezvous technique. World J Gastroenterol 2016;22:2366-2372.

7. Boyce HW, Estores DS, Gaziano J, Padhya T, Runk J. Endoscopic lumen restoration for obstructive aphagia: outcomes of a 25 -year experience. Gastrointest Endosc 2012;76:25-31.

8. Cavell LK, Wong RJ, Shike M, et al. Tu1574 Long term outcomes after endoscopic restoration of complete cervical esophageal obstruction. Gastrointest Endosc 2012;75:AB451-AB452.

9. Dellon ES, Cullen NR, Madanick RD, et al. Outcomes of a combined antegrade and retrograde approach for dilatation of radiation-induced esophageal strictures (with video). Gastrointest Endosc 2010;71:1122-1129.

10. Fowlkes J, Zald PB, Andersen P. Management of complete esophageal stricture after treatment of head and neck cancer using combined anterograde retrograde esophageal dilation. Head Neck 2012;34:821-825.

11. Garcia A, Flores RM, Schattner M, et al. Endoscopic retrograde dilation of completely occlusive esophageal strictures. Ann Thorac Surg 2006;82:1240-1243.

12. Gavriel H, Duong C, Spillane J, Sizeland A. Bidirectional esophageal dilatation in pharyngoesophageal stenosis postradiotherapy. Head Neck 2013;35:733-737.

13. Goguen LA, Norris CM, Jaklitsch MT, et al. Combined antegrade and retrograde esophageal dilation for head and neck cancer-related complete esophageal stenosis. Laryngoscope 2010;120:261-266.

14. Grooteman KV, Wong Kee Song LM, Vleggaar FP, Siersema PD, Baron TH. Functional outcome of patients treated for radiationinduced complete esophageal obstruction after successful endoscopic recanalization (with video). Gastrointest Endosc 2014;80:175-181.

15. Lew RJ, Shah JN, Chalian A, Weber RS, Williams NN, Kochman ML. Technique of endoscopic retrograde puncture and dilatation of total esophageal stenosis in patients with radiationinduced strictures. Head Neck 2004;26:179-183.

16. Malliaris SD, Nemechek AJ, Bulat R, Jaffe BM. Double endoscopic technique for operative dilation of esophageal strictures resistant to conventional therapy. J La State Med Soc 2007;159:159-163, 165.

17. Maple JT, Petersen BT, Baron TH, Kasperbauer JL, Wong Kee Song LM, Larson MV. Endoscopic management of radiation-induced complete upper esophageal obstruction with an antegrade-retrograde rendezvous technique. Gastrointest Endosc 2006;64:822-828.

18. Moyer MT, Stack BC Jr, Mathew A. Successful recovery of esophageal patency in 2 patients with complete obstruction by using combined antegrade retrograde dilation procedure, needle knife, and EUS needle. Gastrointest Endosc 2006;64:789-792.

19. Perbtani Y, Suarez AL, Wagh MS. Emerging techniques and efficacy of endoscopic esophageal reconstruction and lumen restoration for complete esophageal obstruction. Endosc Int Open 2016;4:E136-E142.

20. Schembre D, Dever JB, Glenn M, Bayles S, Brandabur J, Kozarek R. Esophageal reconstitution by simultaneous antegrade/retrograde endoscopy: re-establishing patency of the completely obstructed esophagus. Endoscopy 2011;43:434-437.

21. Soares PC, Bouayed S, Dulguerov P, Frossard JL. Recurrent complete pharyngo-oesophageal stricture treated by multidisciplinary anterograde-retrograde endoscopic dilation. Case Rep Gastroenterol 2016;10:560-567.

22. Steele NP, Tokayer A, Smith RV. Retrograde endoscopic balloon dilation of chemotherapy- and radiation-induced esophageal stenosis under direct visualization. Am JOtolaryngol 2007;28:98-102.

23. van Twisk JJ, Brummer RJ, Manni JJ. Retrograde approach to pharyngoesophageal obstruction. Gastrointest Endosc 1998;48:296-299.

24. Moher D, Liberati A, Tetzlaff J, Altman DG; PRISMA Group. Preferred reporting items for systematic reviews and meta-analyses: the PRISMA statement. Ann Intern Med 2009;151:264-269, W64.

25. Stang A. Critical evaluation of the Newcastle-Ottawa scale for the assessment of the quality of nonrandomized studies in metaanalyses. Eur J Epidemiol 2010;25:603-605.

26. DerSimonian R, Laird N. Meta-analysis in clinical trials. Control Clin Trials 1986;7:177-188.

27. Sutton AJ, Abrams KR, Jones DR, et al. Methods for meta-analysis in medical research. John Wiley \& Sons Ltd.: New York; 2000, pp. 205-228.

28. Kanwal F, White D. Systematic reviews and meta-analyses in clinical gastroenterology and hepatology. Clin Gastroenterol Hepatol 2012;10:1184-1186.

29. Higgins JP, Thompson SG, Deeks JJ, Altman DG. Measuring inconsistency in meta-analyses. BMJ 2003;327:557-560.

30. Guyatt GH, Oxman AD, Kunz R, et al. GRADE guidelines: 7. Rating the quality of evidence inconsistency. $J$ Clin Epidemiol 2011;64:1294-1302.

31. Riley RD, Higgins JP, Deeks JJ. Interpretation of random effects meta-analyses. BMJ 2011;342:d549.

32. Easterbrook PJ, Berlin JA, Gopalan R, Matthews DR. Publication bias in clinical research. Lancet 1991;337:867-872.

33. Wagh MS, Yang D, Chavalitdhamrong D, Draganov PV. Per-oral endoscopic tunneling for restoration of the esophagus (POETRE). Gastrointest Endosc 2014;80:330.

34. Fuccio L, Hassan C, Frazzoni L, Miglio R, Repici A. Clinical outcomes following stent placement in refractory benign esophageal stricture: a systematic review and meta-analysis. Endoscopy 2016;48:141-148.

35. Repici A, Small AJ, Mendelson A, et al. Natural history and management of refractory benign esophageal strictures. Gastrointest Endosc 2016;84:222-228.

36. Walter D, van den Berg MW, Hirdes MM, et al. Dilation or biodegradable stent placement for recurrent benign esophageal strictures: a randomized controlled trial. Endoscopy 2018;50:1146 1155 . 


\section{Supplementary Figure}

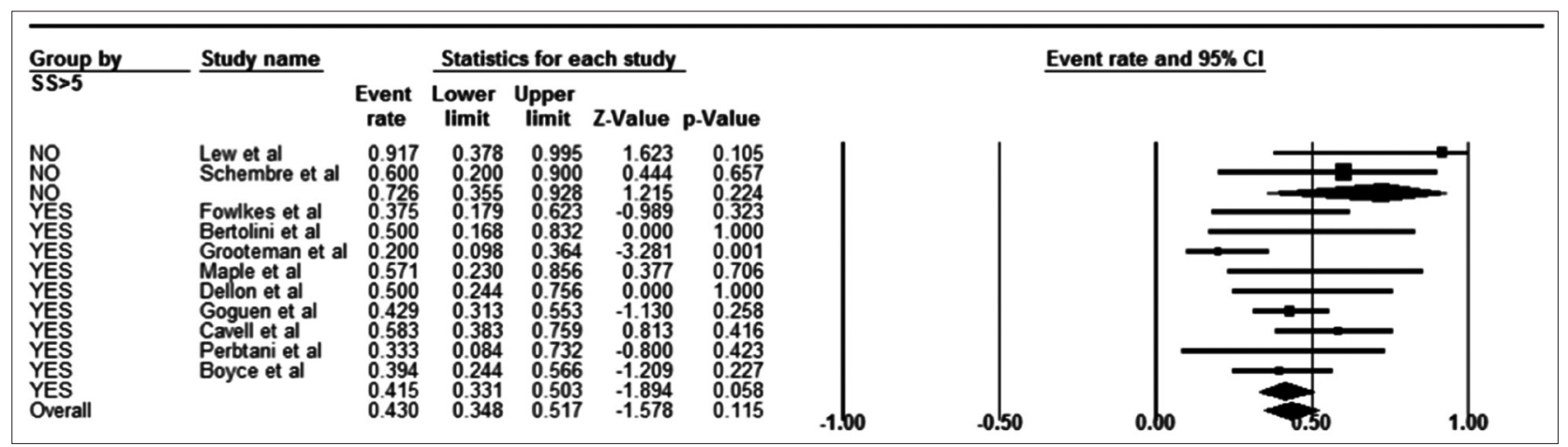

Supplementary Figure 1 Forest plot. Free of percutaneous endoscopic gastrostomy tube by sample size (SS) $<5$ patients and $>5$ patients CI, confidence interval

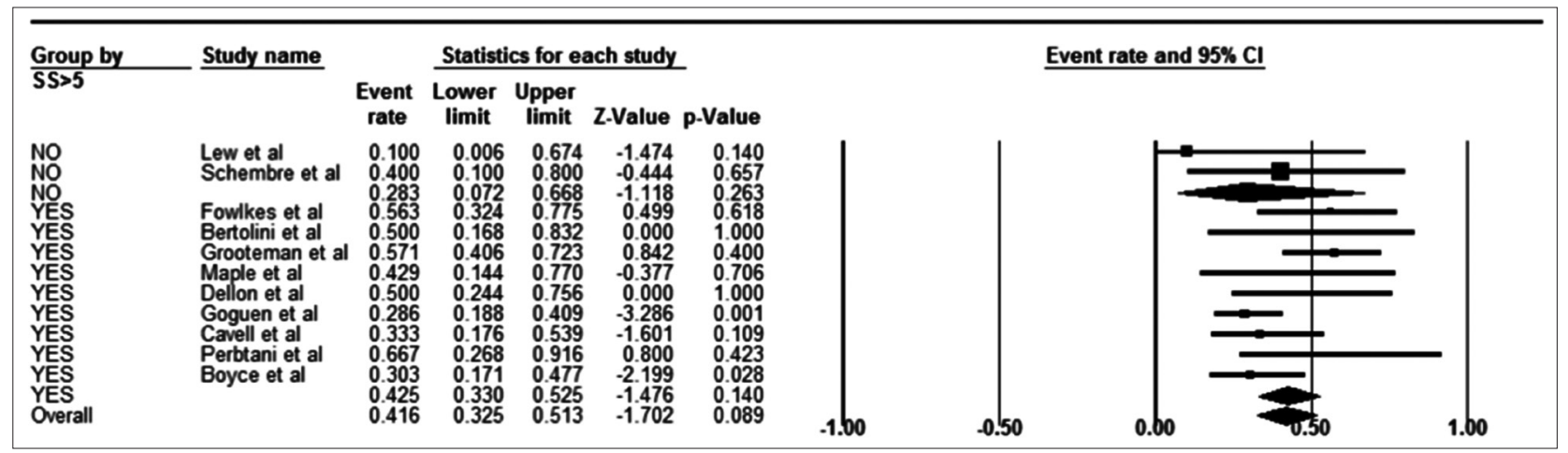

Supplementary Figure 2 Forest plot. percutaneous endoscopic gastrostomy tube dependency by sample size (SS) $<5$ patients and $>5$ patients CI, confidence interval

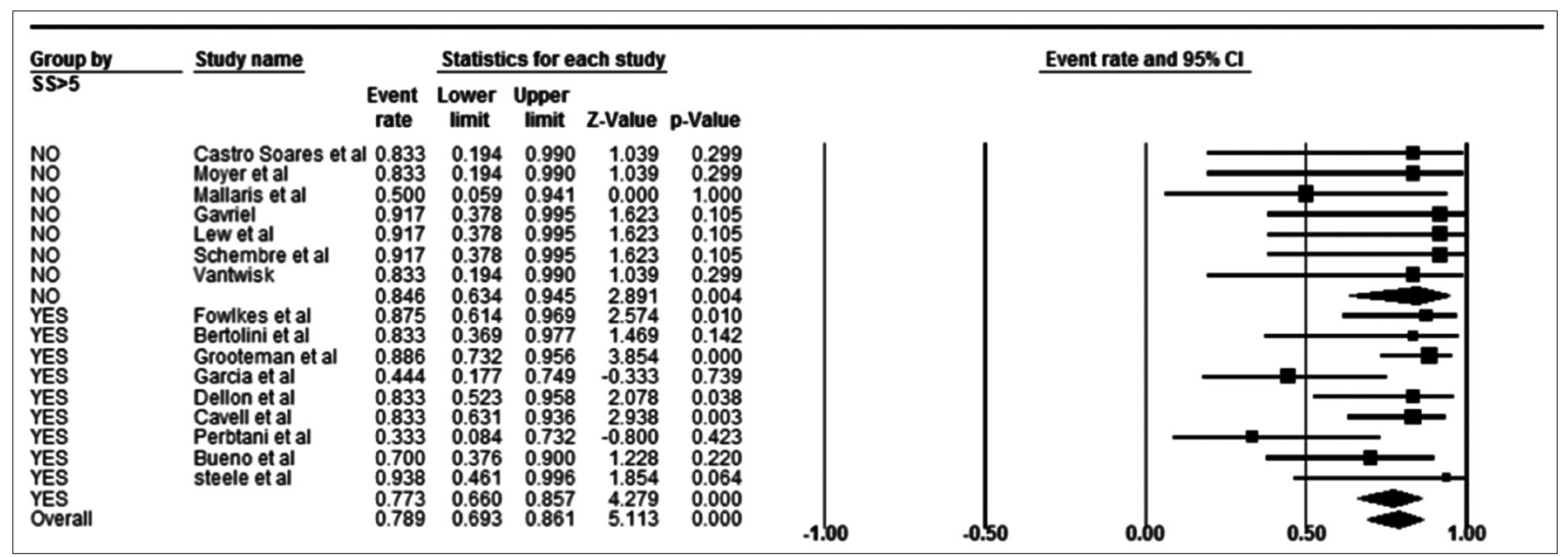

Supplementary Figure 3 Forest plot. Repeat dilation by sample size $(\mathrm{SS})<5$ patients and $>5$ patients

CI, confidence interval 


\begin{tabular}{|c|c|c|c|c|c|c|c|c|c|}
\hline \multirow{2}{*}{$\frac{\text { Group by }}{\text { SS }>5}$} & \multirow[t]{2}{*}{ Study name } & \multicolumn{5}{|c|}{ Statistics for each study } & \multicolumn{3}{|c|}{ Event rate and $95 \% \mathrm{Cl}$} \\
\hline & & $\begin{array}{c}\text { Event } \\
\text { rate }\end{array}$ & $\begin{array}{c}\text { Lower } \\
\text { limit }\end{array}$ & $\begin{array}{l}\text { Upper } \\
\text { limit }\end{array}$ & Z-Value & p-Value & & & \\
\hline $\begin{array}{l}\text { NO } \\
\text { NO } \\
\text { NO } \\
\text { NO } \\
\text { NO } \\
\text { NO } \\
\text { YES } \\
\text { YES } \\
\text { YES } \\
\text { YES } \\
\text { YES } \\
\text { YES } \\
\text { YES } \\
\text { YES } \\
\text { YES } \\
\text { YES } \\
\text { YES } \\
\text { YES } \\
\text { Overall }\end{array}$ & $\begin{array}{l}\text { Mallaris et al } \\
\text { Gaviel } \\
\text { Lew et al } \\
\text { Schembre et al } \\
\text { Vantwisk } \\
\text { Fowlkes et al } \\
\text { Bertolini et al } \\
\text { Grooteman et al } \\
\text { Garcia et al } \\
\text { Maple et al } \\
\text { Dellon et al } \\
\text { Goguen et al } \\
\text { Cavell et al } \\
\text { Perbani et al } \\
\text { Bueno et al } \\
\text { steele et al }\end{array}$ & $\begin{array}{l}0.167 \\
0.083 \\
0.083 \\
0.083 \\
0.167 \\
0.09 \\
0.029 \\
0.071 \\
0.086 \\
0.050 \\
0.286 \\
0.083 \\
0.032 \\
0.020 \\
0.071 \\
0.045 \\
0.143 \\
0.075 \\
0.080\end{array}$ & $\begin{array}{l}0.010 \\
0.005 \\
0.005 \\
0.005 \\
0.010 \\
0.032 \\
0.002 \\
0.004 \\
0.028 \\
0.003 \\
0.072 \\
0.012 \\
0.008 \\
0.001 \\
0.004 \\
0.003 \\
0.020 \\
0.043 \\
0.048\end{array}$ & $\begin{array}{l}0.806 \\
0.622 \\
0.622 \\
0.622 \\
0.806 \\
0.314 \\
0.336 \\
0.577 \\
0.234 \\
0.475 \\
0.673 \\
0.413 \\
0.118 \\
0.251 \\
0.577 \\
0.448 \\
0.581 \\
0.128 \\
0.130\end{array}$ & $\begin{array}{l}-1.039 \\
-1.623 \\
-1.623 \\
-1.623 \\
-1.039 \\
-3.121 \\
-2.436 \\
-1.748 \\
-3.920 \\
-2.029 \\
-1.095 \\
-2.296 \\
-4.756 \\
-2.724 \\
-1.748 \\
-2.103 \\
-1.659 \\
-8.237 \\
-8.791\end{array}$ & $\begin{array}{l}0.299 \\
0.105 \\
0.105 \\
0.105 \\
0.299 \\
0.002 \\
0.015 \\
0.081 \\
0.000 \\
0.042 \\
0.273 \\
0.022 \\
0.000 \\
0.006 \\
0.081 \\
0.035 \\
0.097 \\
0.000 \\
0.000\end{array}$ & -1.60 & -0.50 & 1.60 \\
\hline
\end{tabular}

Supplementary Figure 4 Forest plot. Perforation by sample size $(S S)<5$ patients and $>5$ patients CI, confidence interval

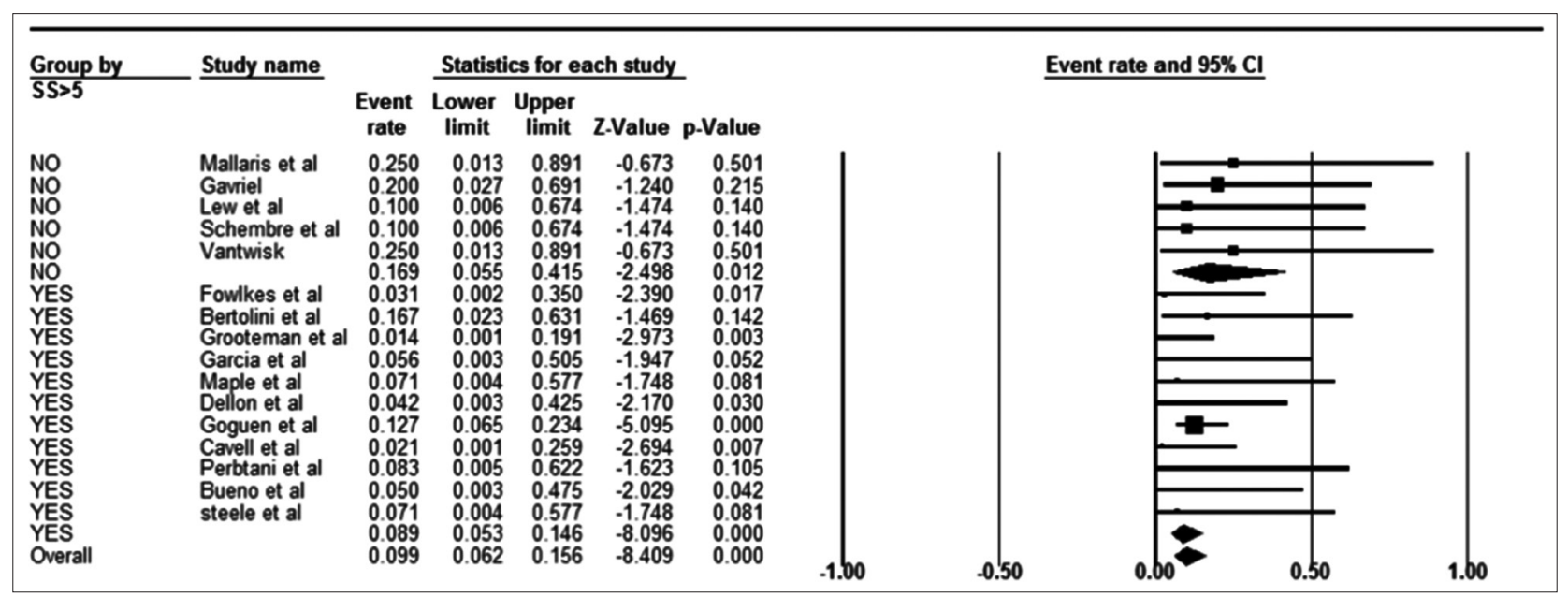

Supplementary Figure 5 Forest plot. Pneumo-mediastinum by sample size (SS) $<5$ patients and $>5$ patients CI, confidence interval

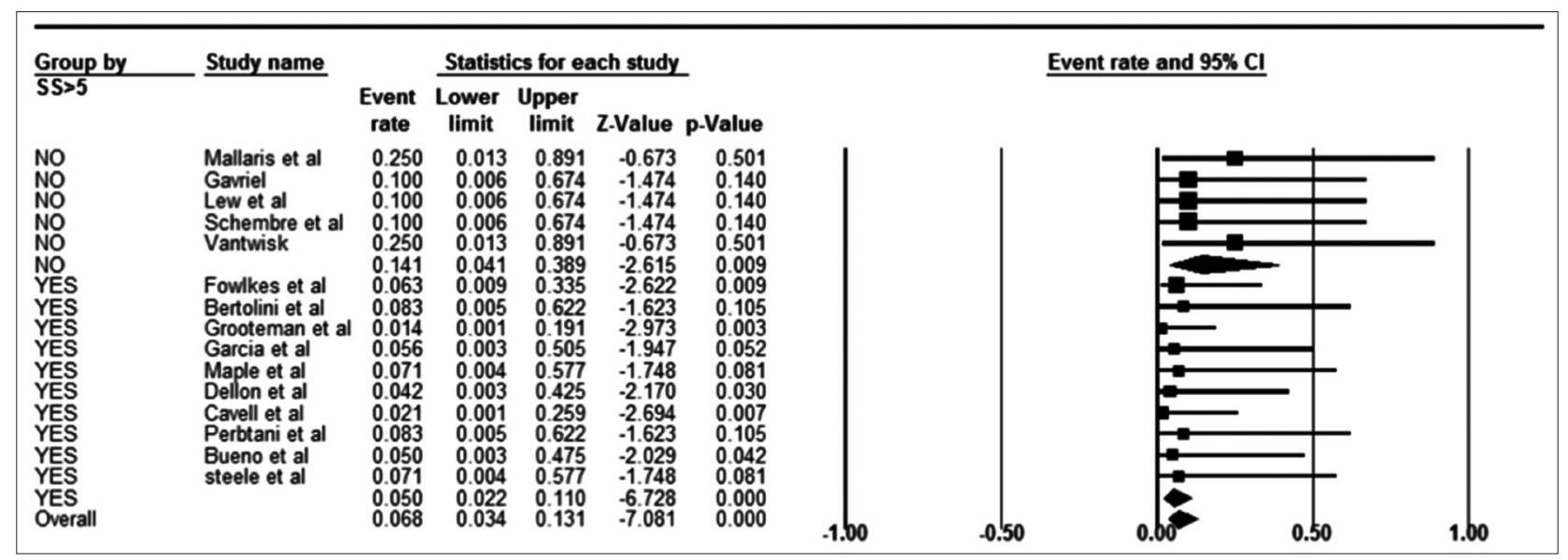

Supplementary Figure 6 Forest plot. Death by sample size (SS) $<5$ patients and $>5$ patients CI, confidence interval 


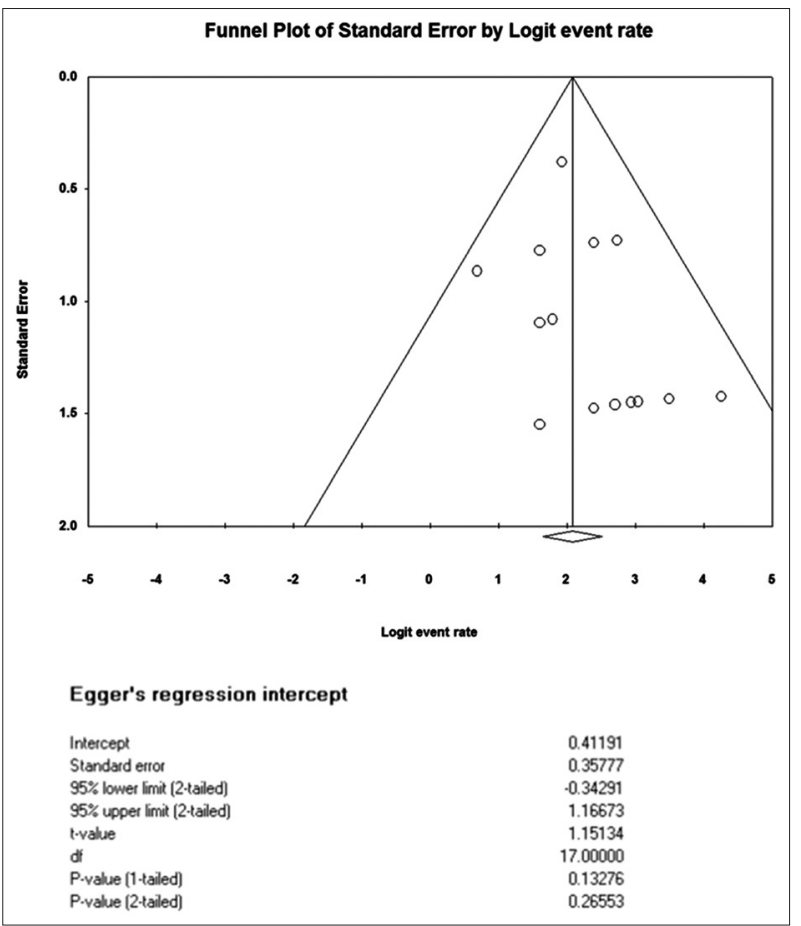

Supplementary Figure 7 Funnel plot and Egger's intercept. All studies 


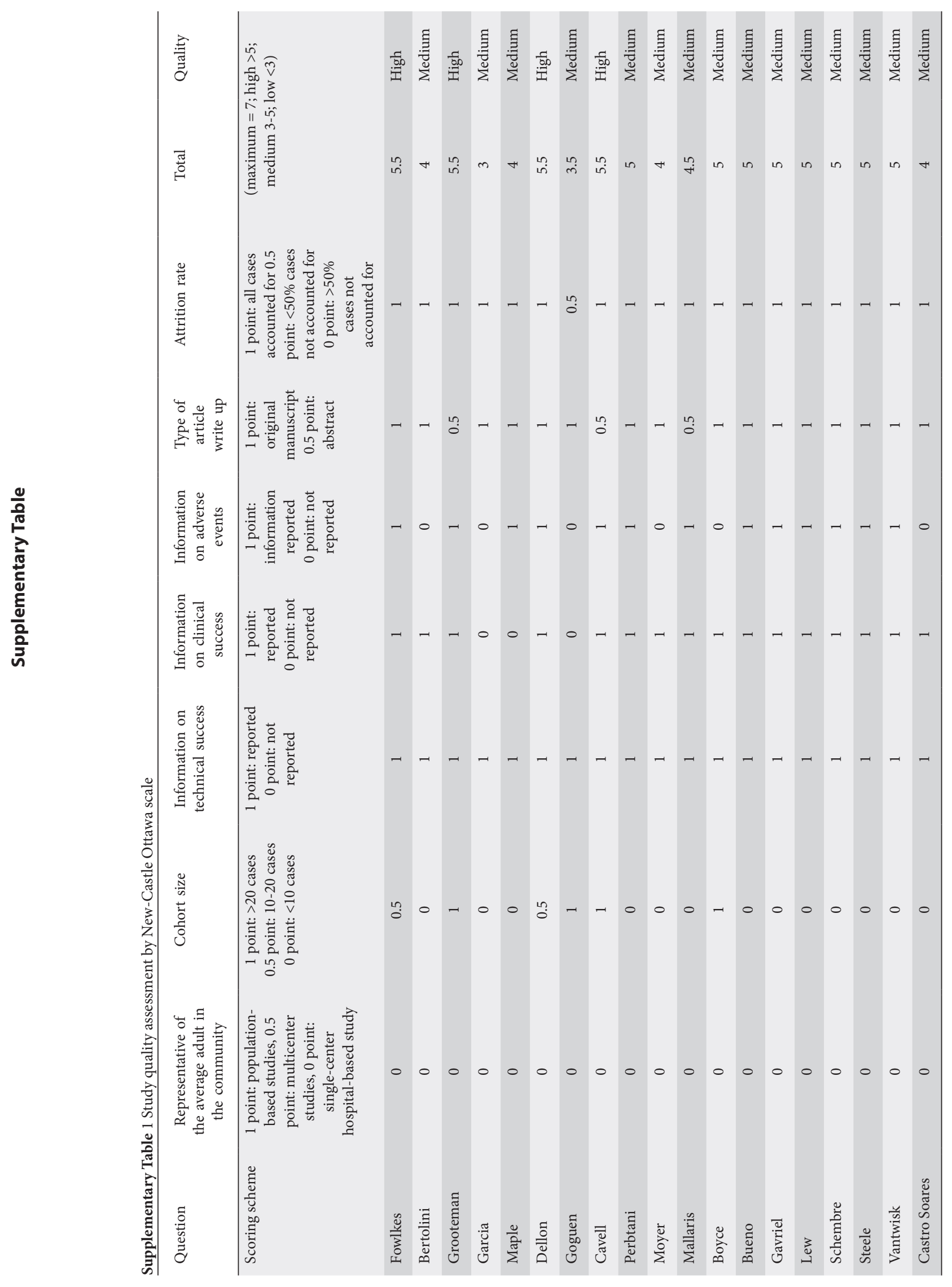

